\title{
Remarks concerning Driver's equation
}

\author{
by Gerd Herzog and Roland Lemmert (Karlsruhe)
}

Abstract. We consider uniqueness for the initial value problem $x^{\prime}=1+f(x)-f(t)$, $x(0)=0$. Several uniqueness criteria are given as well as an example of non-uniqueness.

Let $f: \mathbb{R} \rightarrow \mathbb{R}$ be continuous. We consider the initial value problem

$$
\left\{\begin{array}{l}
x^{\prime}(t)=1+f(x(t))-f(t), \quad t \geq 0, \\
x(0)=0
\end{array}\right.
$$

which has $x(t)=t$ as a solution. Driver [1] asks whether this is in general the only one and proves [2]:

Proposition 1. There is no solution $x$ with $x(t)<t(t>0)$ and $x^{\prime}(t)$ decreasing and no solution $x(t)>t(t>0)$ with $x^{\prime}(t)$ increasing (in the wider sense).

( $f$ is to be substituted by $-g$ in Driver's terminology.)

Nowak [3] remarks that Driver's question is not completely answered yet.

We will sharpen Proposition 1 in several ways and give examples of continuous functions $f$ such that (1) is not uniquely solvable. We also provide conditions on $f$ such that (1) is uniquely solvable.

We begin with

Proposition 2. For each solution $x$ of (1) we have $x(t) \leq t, t \geq 0$.

Proof. We rewrite the differential equation as

$$
\exp \left(x^{\prime}(t)-1\right)=\exp (f(x(t))) \cdot \exp (-f(t)),
$$

and because $\exp (s-1) \geq s(s \in \mathbb{R})$ we get

$$
x^{\prime}(t) \exp (-f(x(t))) \leq \exp (-f(t))
$$

1991 Mathematics Subject Classification: 34A34, 34A40.

Key words and phrases: ordinary differential equations, uniqueness conditions. 
and by integration

$$
\int_{0}^{x(t)} \exp (-f(s)) d s \leq \int_{0}^{t} \exp (-f(s)) d s
$$

which gives $x(t) \leq t, t \geq 0$.

Another way of looking at this problem is the following:

The initial value problem

$$
\left\{\begin{array}{l}
y^{\prime}(t)=\exp (f(y(t))) \cdot \exp (-f(t)) \\
y(0)=0
\end{array}\right.
$$

is uniquely solvable, since it has separated variables with $\exp (f(0)) \neq 0$; its solution is $y(t)=t$, and any solution of (1) is a subsolution to (2), hence $x(t) \leq t$.

We next give a necessary condition which solutions of (1) have to satisfy.

Proposition 3. Let $d(t)=t-x(t), x$ any solution of $(1)$, denote by $\sigma_{f}(t)$ the oscillation of $f$ over the interval $[0, t]$, and let $(\cdot)_{+}$be the positive part of a function. Then

$$
0 \leq d(t) \leq \sigma_{f}(t) \int_{0}^{t}\left(d^{\prime}(s)\right)_{+} d s, \quad t \geq 0 .
$$

Proof. Let $t>0$ and $c$ a constant which will be determined later. Then $d$ satisfies

$$
d^{\prime}(t)=-(f(x(t))-c) d^{\prime}(t)+f(t)-c-(f(x(t))-c) x^{\prime}(t) .
$$

By integration we get

$$
\int_{x(t)}^{t}(1-(f(s)-c)) d s=\int_{0}^{t}(f(x(s))-c)\left(x^{\prime}(s)-1\right) d s .
$$

(This relation is most easily verified by differentiation.)

Now setting $c=\min \{f(s): 0 \leq s \leq t\}$ we have

$$
\sigma_{f}(t) \geq f(s)-c \geq 0, \quad \sigma_{f}(t) \geq f(x(s))-c \geq 0
$$

and therefore

$$
(t-x(t))\left(1-\sigma_{f}(t)\right) \leq \sigma_{f}(t) \int_{0}^{t}\left(-d^{\prime}(s)\right)_{+} d s,
$$

from which (3) easily follows by using $t-x(t)=\int_{0}^{t} d^{\prime}(s) d s$.

Proposition 3 shows that (1) cannot have a solution different from $t$ near 0 such that $x^{\prime}(t) \leq 1$ (which in particular holds if $x^{\prime}$ decreases since $\left.x^{\prime}(0)=1\right)$ : In this case $\left(d^{\prime}(s)\right)_{+}=d^{\prime}(s)$, which implies $d \equiv 0$ for small $t>0$ (i.e., for those $t$ with $\sigma_{f}(t)<1$ ). 
Now fix $t_{0}>0$ and assume there is a solution of (1) such that $x\left(t_{0}\right)=t_{0}$. Then $z(t)=x\left(t+t_{0}\right)-t_{0}$ satisfies

$$
\left\{\begin{array}{l}
z^{\prime}(t)=1+g(z(t))-g(t) \\
z(0)=0
\end{array}\right.
$$

where $g$ is defined by $g(s)=f\left(s+t_{0}\right)-t_{0}$. By Proposition 3 we have

$$
0 \leq t-z(t) \leq \sigma_{g}(t) \int_{0}^{t}\left(1-z^{\prime}(s)\right)_{+} d s, \quad t \geq 0,
$$

from which we get

$$
0 \leq t-x(t) \leq \sigma_{f}\left(t_{0}, t\right) \int_{t_{0}}^{t}\left(1-x^{\prime}(s)\right)_{+} d s, \quad t \geq t_{0},
$$

where $\sigma_{f}\left(t_{0}, t\right)$ denotes the oscillation of $f$ over the interval $\left[t_{0}, t\right]$. This shows that no solution can leave the diagonal at a time $t_{0}>0$ if $x^{\prime}(t) \leq 1$.

Of course, if $f$ is decreasing, (1) is uniquely solvable by standard uniqueness theorems. On the other hand, Proposition 3 implies uniqueness if $f$ is increasing; for in this case we have the inequality $x^{\prime}(t) \leq 1, t \geq 0$, because of $x(t) \leq t(t \geq 0)$. Remarkably enough, from the above considerations we see that if $f$ is locally of bounded variation, then (1) is uniquely solvable: We write $f=f_{1}-f_{2}, f_{1}, f_{2}$ increasing, and get

$$
x^{\prime}(t) \geq 1+f_{1}(x(t))-f_{1}(t), \quad t \geq 0,
$$

so $x(t) \geq t$, and finally $x(t)=t, t \geq 0$.

Proposition 4. Let $f^{\prime}(s)$ exist for $s>0$ and let there exist $c<\bar{c}<1$ such that

$$
f^{\prime}(s) \leq \frac{c}{s}+1-\bar{c}, \quad s \in(0,1] .
$$

Then problem (1) is uniquely solvable in $[0,1]$.

Proof. From $1-x^{\prime}(t)=-f(x(t))+f(t)$ we get for $t, x(t)>0$,

$$
1-x^{\prime}(t)=\int_{x(t)}^{t} f^{\prime}(s) d s \leq(1-\bar{c})(t-x(t))+c(\log t-\log x(t))
$$

or

$$
x^{\prime}(t) \geq 1+c \log x(t)-c \log t+(1-\bar{c})(x(t)-t) .
$$

If (1) is not uniquely solvable, we may assume by Kneser's theorem that there is a solution $x:[0,1] \rightarrow \mathbb{R}$, positive in $(0,1]$, such that $1>x(1)>d>0$, with $d$ to be determined in a moment.

We now consider the initial value problem

$$
\left\{\begin{array}{l}
z^{\prime}(t)=1+c \log z(t)-c \log t+(1-\bar{c})(z(t)-t), \\
z(1)=x(1)
\end{array}\right.
$$


By (4), $x$ is a subsolution to the left for $(5)$ in $(0,1]$. Now, because of $c<$ $\bar{c}<1$, there is $1>d>0$ such that

$$
\bar{c}(s-1) \leq c \log s, \quad d \leq s \leq 1,
$$

so that $y(t)=x(1) \cdot t$ satisfies

$$
y^{\prime}(t) \leq 1+c \log y(t)-c \log t+(1-\bar{c})(y(t)-t), \quad 0<t \leq 1 .
$$

Therefore $y$ is a supersolution of (5) to the left and by standard comparison theorems we get

$$
x(t) \leq y(t), \quad t \in(0,1] .
$$

But then $x$ cannot be a solution of $(1)$, since (6) implies $x^{\prime}(0) \leq x(1)<1$.

Proposition 5. If $f$ satisfies

$$
f(t)-f(x) \leq \frac{1}{t}(t-x), \quad 0 \leq x<t \leq 1,
$$

then (1) is uniquely solvable in $[0,1]$.

Proof. The proof follows the same ideas as the proof of Proposition 4. In this case we write

$$
1-x^{\prime}(t)=f(t)-f(x(t)) \leq \frac{1}{t}(t-x(t)), \quad 0<t \leq 1 .
$$

Then $x$ is a subsolution to the left for

$$
z^{\prime}(t)=\frac{1}{t} z(t), \quad z(1)=x(1) .
$$

Standard comparison theorems [4] give $x(t) \leq x(1) \cdot t, 0<t \leq 1$, since $z(t)=x(1) \cdot t$ is the solution to this latter problem. Hence again $x^{\prime}(0) \leq x(1)$, which is impossible if $x(1)<1$.

We finally construct an example of a bounded continuous function $f$ : $\mathbb{R} \rightarrow \mathbb{R}$ such that (1) is not uniquely solvable.

To this end we define by induction a sequence $b_{1}=1, a_{1}, b_{2}, a_{2}, b_{3}, \ldots$ of numbers which tends strictly monotonically to zero; $f$ will be zero outside $(0,1)$ and on any interval $\left[b_{n+1}, a_{n}\right](n \geq 1)$, and positive elsewhere.

Let $b_{1}=1>a_{1}>0$ (the value of $a_{1}$ will be fixed later), $\gamma:[0,1] \rightarrow$ [0,1] continuous, $\gamma(0)=\gamma(1)=0$ and $m=\int_{0}^{1} \gamma(s) d s>0$. We define $\gamma_{1}(s)=\gamma\left(\left(s-a_{1}\right) /\left(1-a_{1}\right)\right), m_{1}=\left(1 /\left(b_{1}-a_{1}\right)\right) \int_{a_{1}}^{b_{1}} \gamma_{1}(s) d s$ and remark that $m_{1}=m$.

By $I(\mu)$ we denote $\int_{0}^{1} d s /(1+\mu \gamma(s))$. Let $\left(\mu_{n}\right)$ be a sequence of positive numbers tending to zero, $\mu_{1}=1, \mu_{n} \leq 1$. 
In $\left[a_{1}, b_{1}\right]$ we solve $x^{\prime}(t)=1-\gamma_{1}(t), x\left(b_{1}\right)=a_{1}$ and set $b_{2}=x\left(a_{1}\right)$, which gives

$$
a_{1}-b_{2}=b_{1}-a_{1}-\int_{a_{1}}^{b_{1}} \gamma_{1}(s) d s
$$

or

$$
a_{1}-b_{2}=(1-m)\left(1-a_{1}\right) .
$$

Therefore $b_{2}<a_{1}$, and we define $f=\gamma_{1}$ in $\left[a_{1}, b_{1}\right], f \equiv 0$ in $\left[b_{2}, a_{1}\right]$.

Next we choose $a_{2}$ such that

$$
b_{2}-a_{2}=\left(a_{1}-b_{2}\right) \cdot \frac{1}{I\left(\mu_{2}\right)}=(1-m)\left(1-a_{1}\right) \cdot \frac{1}{I\left(\mu_{2}\right)}
$$

and solve $x^{\prime}(t)=1+\gamma_{2}(x(t)), x\left(a_{1}\right)=b_{2}$, where

$$
f(s):=\gamma_{2}(s):=\mu_{2} \gamma_{1}\left(\frac{b_{1}-a_{1}}{b_{2}-a_{2}}\left(s-a_{2}\right)+a_{1}\right), \quad a_{2} \leq s \leq b_{2} .
$$

Since the differential equation for $x$ has separated variables, an easy calculation shows $a_{2}=x\left(b_{2}\right)$.

Up to now $f$ is defined on $\left[a_{2}, 1\right]$, and $x$ satisfies the differential equation from (1) on $\left[a_{2}, 1\right], x$ being increasing with values in $\left[a_{2}, 1\right]$.

To proceed by induction, let $a_{n}<b_{n}$ be defined,

$$
f(t):=\gamma_{n}(t)=\mu_{n} \gamma\left(\frac{1}{b_{n}-a_{n}}\left(t-a_{n}\right)\right), \quad a_{n} \leq t \leq b_{n} .
$$

We solve $x^{\prime}(t)=1-\gamma_{n}(t), t \in\left[a_{n}, b_{n}\right], x\left(b_{n}\right)=a_{n}$, set $b_{n+1}=x\left(a_{n}\right)<a_{n}$, define $f \equiv 0$ in $\left[b_{n+1}, a_{n}\right]$ and $a_{n+1}$ by

$$
I\left(\mu_{n+1}\right)\left(b_{n+1}-a_{n+1}\right)=a_{n}-b_{n+1},
$$

so $b_{n+1}>a_{n+1}$. Now we solve $x^{\prime}(t)=1+\gamma_{n+1}(x(t)), x\left(a_{n}\right)=b_{n+1}$ on $\left[a_{n+1}, b_{n+1}\right]$, the solution of which satisfies $x\left(b_{n+1}\right)=a_{n+1}, f$ is defined on $\left[a_{n+1}, 1\right]$ and $x$ satisfies the differential equation in (1).

By our construction we have, for $n \geq 1$,

$$
\begin{aligned}
a_{n}-b_{n+1} & =\left(1-\mu_{n} m\right)\left(b_{n}-a_{n}\right), \\
b_{n+1}-a_{n+1} & =\left(1-\mu_{n} m\right)\left(b_{n}-a_{n}\right) \cdot \frac{1}{I\left(\mu_{n+1}\right)},
\end{aligned}
$$

so for $n \geq 2$,

$$
\begin{gathered}
b_{n}-a_{n}=\left(1-a_{1}\right)(1-m) \cdot \prod_{k=2}^{n-1}\left(1-\mu_{k} m\right) \cdot \prod_{k=2}^{n} \frac{1}{I\left(\mu_{k}\right)}, \\
a_{n-1}-b_{n}=\left(1-a_{1}\right)(1-m) \cdot \prod_{k=2}^{n-1}\left(1-\mu_{k} m\right) \cdot \prod_{k=2}^{n-1} \frac{1}{I\left(\mu_{k}\right)} .
\end{gathered}
$$


From Jensen's inequality, applied to the convex functions $h(x)=1 /(1+\mu x)$ $(\mu \geq 0, x \geq 0)$, we have

$$
I(\mu)=\frac{1}{b_{1}-a_{1}} \int_{a_{1}}^{b_{1}} \frac{d s}{1+\mu \gamma_{1}(s)} \geq \frac{1}{1+\mu m} ;
$$

therefore the sequence $b_{1}, a_{1}, b_{2}, a_{2}, b_{3}, a_{3}, \ldots$ is convergent if

$$
\sum_{n=3}^{\infty} \prod_{k=2}^{n-1}\left(1-\mu_{k}^{2} m^{2}\right)
$$

converges, which is the case, for example, for $\mu_{k}=1 / \sqrt[4]{k}, k \in \mathbb{N}$, as is easily verified using Raabe's test for convergence. For suitable $a_{1}<1$ we finally get $\lim _{n \rightarrow \infty} a_{n}=\lim _{n \rightarrow \infty} b_{n}=0$. So $f$ is defined everywhere, continuous, bounded, and the solution $x$ solves (1) with $x(t)<t, t \in(0,1]$.

R e marks. 1) For a suitable choice of $\gamma$, the function $f$ is $C^{\infty}$ in $\mathbb{R} \backslash\{0\}$.

2 ) If we define $F(t, x)=1+f(x)-f(t)$, Kamke's or related uniqueness theorems are of course applicable if $f$ satisfies an appropriate condition. Our condition in Proposition 4 cannot be subsumed under this, since, for an autonomous equation $x^{\prime}=g(x)$, the condition $g^{\prime}(x) \leq c / x$ does not imply uniqueness, as $g(x)=\sqrt{x}$ shows.

3 ) It would be interesting to know whether the condition $f^{\prime}(t) \leq 1 / t+1$, $0<t \leq 1$, implies uniqueness for (1). This condition would contain the conditions of Propositions 4 and 5.

\section{References}

[1] R. D. Driver, Advanced problem 5415, Amer. Math. Monthly 73 (1966), 783.

[2] R. D. Driver, D. W. Sasser and R. J. Thompson, Solutions of advanced problems, ibid. 76 (1969), 948-949.

[3] Ch. Nowak, Eindeutigkeit und Nichteindeutigkeit bei gewöhnlichen Differentialgleichungen, Habilitationsschrift, Universität Klagenfurt, 1990.

[4] W. Walter, Gewöhnliche Differentialgleichungen, 4. Auflage, Springer, Berlin, 1990.

MATHEMATISCHES INSTITUT I

UNIVERSITÄT KARLSRUHE

ENGLERSTR. 2

POSTFACH 6380

D-76128 KARLSRUHE 1, GERMANY 